\title{
Adaptive Neural Network Filter for Visual Evoked Potential Estimation
}

\author{
K.S.M.Fung ${ }^{1}$, F.K.Lam ${ }^{2}$, F.H.Y.Chan ${ }^{3}$, P.W.F.Poon ${ }^{4}$, J.G.Liu ${ }^{5}$ \\ 1,2,3,5 Department of Electrical and Electronic Engineering, The University of Hong Kong, Hong Kong \\ ${ }^{4}$ Department of Physiology, School of Medicine, National Cheng Kung University, Tainan, Taiwan \\ 'smfung@hkueee.hku.hk, ${ }^{2}$ fklam@hkueee.hku.hk, ${ }^{3}$ fhychan@hkueee.hku.hk, \\ 4ppoon@mail.ncku.edu.tw, ${ }^{4}$ jgliu@hkueee.hku.hk
}

\begin{abstract}
We describe a new approach to enhance the signal-to-noise-ratio (SNR) of visual evoked potential (VEP) based on an adaptive neural network filter. Neural networks are usually used in an non-adaptive way. The weights in the neural network are adjusted during training but remain constant in actual use. In this paper, we use an adaptive neural network filter with adaptation capabilities similar to those of the traditional linear adaptive filter, and suitable training scheme is also examined. In contrast with linear adaptive filters, adaptive neural network filters possess non-linear characteristics which can better match the non-linear behaviour of evoked potential signals. Simulations employing VEP signals obtained experimentally confirm the superior performance of the adaptive neural network filter against traditional linear adaptive filter.
\end{abstract}

Keywords: Adaptive Neural Network Filter, Visual Evoked Potential, Ensemble Average, Linear Adaptive Filter

\section{Introduction}

Visual Evoked Potential (VEP) is the gross electrical response of the nervous system to visual stimulation, say, a flash of light. It is of special interest in many clinical applications including the diagnosis of neurological disorders $[1-2]$. The VEP is usually immersed in the ongoing background electroencephalogram (EEG) with the SNR well below $0 \mathrm{~dB}$, making the estimation of VEP a difficult task. So far, many methods have been utilized to estimate the evoked potentials. Among them ensemble averaging (EA) is commonly employed to improve SNR in evoked potential monitoring. EA is based on the assumption that the underlying signal evoked by the stimulation in each trial is the same, and that the background EEG activity is random and uncorrelated with the EP. However, in practice, these assumptions may not be valid because single trial EP is not a signal whose components are deterministically related to the stimulus, but is made up of components that may shift in both amplitude and latency from one stimulus application to the next [4] - [6]. Due to the poor SNR of raw VEP signal, usually about one hundred ensembles are required for VEP measurement. As a result the averaged signal tends to smear or smooth any variations from ensemble to ensemble. It is impossible to study the variation of the underlying signal across trials. Therefore it is desired to investigate new methods to enhance the SNR of VEP signal in order to reduce the number of ensembles required in VEP acquisition.
In recent years, many researchers have described a variety of approaches to extract the evoked potential (EP) from the background ongoing EEG [6 - 8]. Among the proposed filtering schemes, linear adaptive filters are widely applied in enhancing SNR of evoked potentials [9-11]. Adaptive filter has the advantage of adapting itself to the non-stationary characteristic of the EP signal. Most of the investigated adaptive filters for EP estimation are linear. However given the possible non-linear nature of EP signal, an adaptive non-linear filter may be expected to have a better performance in enhancing the SNR of EP signal.

Artificial neural networks have been utilized as filters. Shirvaikar and Trivedi described a neural network filter to extract weak targets in heavy noisy background [12]. Acoustic signals emitted by surface and submarine targets are separated by a trained neural network [13]. Artificial neural network filters with non-linear transfer functions have been applied to the estimation of VEP [14]. To cater for the nonstationary characteristic of EP signals, an adaptive version of artificial neural network filter has been implemented in this paper.

\section{The Basics of Neural Network Filter}

We have designed a neural network filter consisting of a feedforward multi-layer-perceptron with one hidden layer. The artificial neural network has 20 input nodes, 20 hidden nodes and 1 output nodes. The 
neurons in any layer are connected only to the neurons in the next layer (Fig. 1). The neuron transfer function takes the form of an non-linear sigmoidal function. Noisy VEP signal, $x$, is sampled at the rate of $f_{s}$. The neural network filter is used as a moving window which scans through the noisy signal and produces a filtered output, $\mathbf{Y}_{\mathbf{k}}=\left[y_{k}\right]$ (where $\mathrm{k}=1$ to

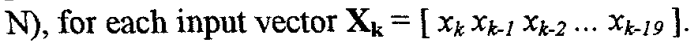

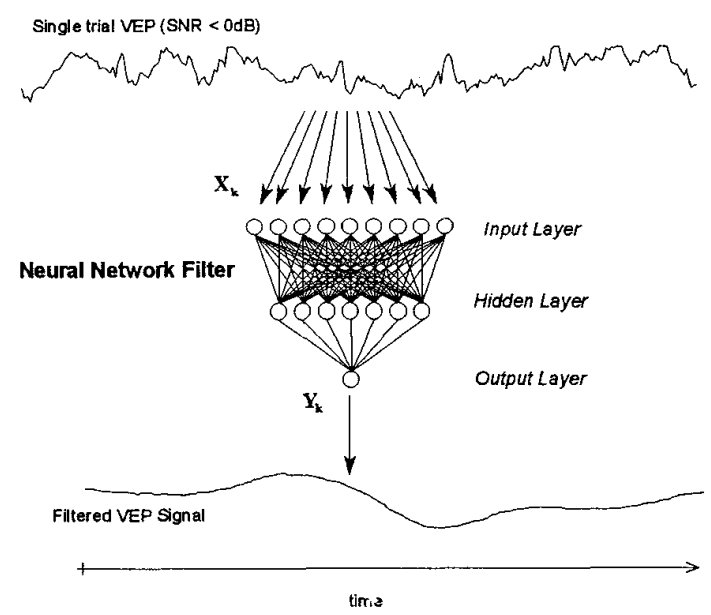

Fig. 1: The schematic of a VEP neural network filter

The training of the neural network filter requires examples of the noisy input signal and the corresponding desired output signal. In the case of VEP filtering, the noisy signal is a single-trial VEP signal and the desired output signal, $d$, is generated by ensemble averaging a number of VEP ensembles. The traditional back-error-propagation training algorithm is applied to train the network. Mean square error (MSE) is the cost function of training, defined as:

$$
M S E=\frac{1}{N} \sum_{k=1}^{N} e_{k}{ }^{2}=\frac{1}{N} \sum_{k=1}^{N}\left(d_{k}-y_{k}\right)^{2}
$$

The training process is aimed at reducing the value of MSE. Thus the connection weights of the network is adjusted to fit the desired input/output characteristic of the VEP filter.

\section{An Adaptive Neural Network Filter}

An adaptive linear filter has been implemented [9] as shown in Fig. 2(a). The filter is capable of tracking non-stationary EP signals. Following this adaptation idea we designed an adaptive version of neural network filter. The primary input signal is the $n^{\text {th }}$ ensemble of VEP signal while the reference signal is the ensemble averaged signal of $(n-m)^{t h}$ ensemble to $(n-1)^{t h}$ ensemble where $m$ is chosen such that the reference signal has a high correlation with the underlying signal in the primary input and a sufficiently high SNR to achieve a satisfactory weights updating process. In the case of VEP estimation, $\mathrm{m}$ is chosen to be 10 . In each iteration, a new data point, $x_{k}$, of the primary signal is inputted into the input layer through the tapped delay line and a corresponding filter output, $y_{k}$, is produced from the neural network filter. The error term is computed by comparing the reference input, $d_{k}$, and the filter output. The connection weights of the filter are then updated by the method of back-error-propagation in the next iteration with the learning equation given by $\Delta w_{i j}=\mu \varepsilon_{j} y_{i}$ where $\mu$ is the convergence parameter, and the error term, $\varepsilon_{j}$, are $d_{j}-y_{j}$ and $\Sigma\left(\varepsilon_{k} w_{k j}\right)$ for the output nodes and hidden nodes respectively. As iterations proceed from sample to sample, the neural network filter adapts to the non-stationary behaviour of the VEP signal and the adaptation takes place at the rate determined by the convergence parameter. Different from most artificial neural network applications, the training of the adaptive neural network filter never stops during the filtering operation.
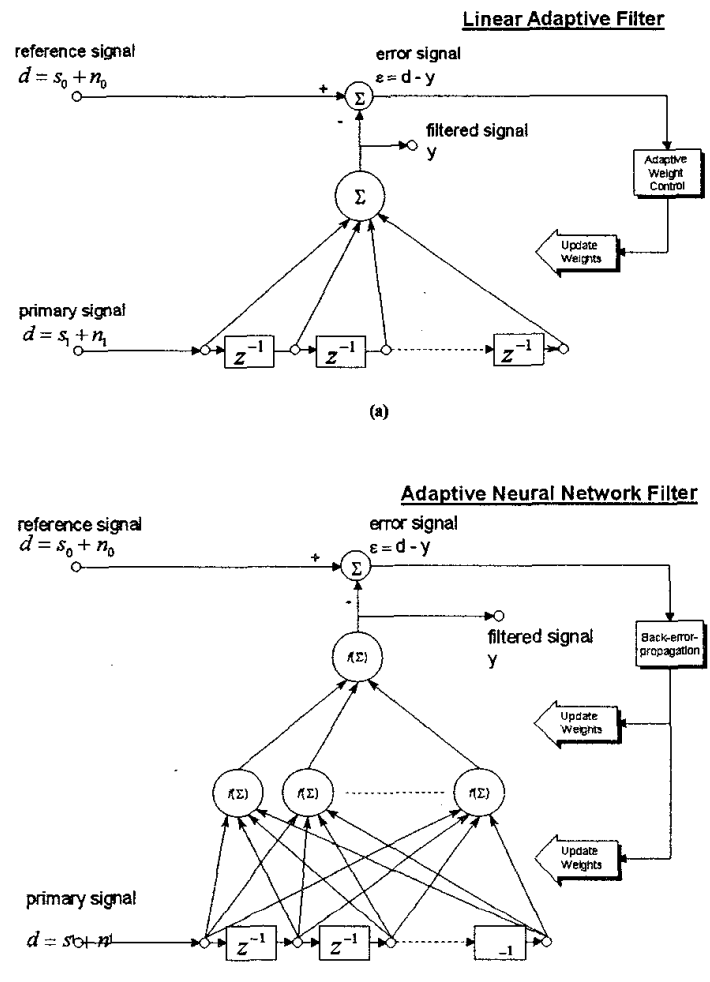

(b)

Fig. 2: (a) The linear adaptive filter and (b) the adaptive neural network filter. The symbol $z^{-1}$ is the unit-delay operator and $f$ is the non-linear transfer function in each neuron. 


\section{Results}

Some simulation results are shown in Fig. 3. The performance of adaptive neural network filter is compared with ensemble averaging. The underlying signal is obtained by ensemble averaging 100 ensembles of VEP signal from an adult. The background ongoing EEG noisy signal is simulated by adding a sequence of random number which has a uniform distribution. A smoothing filter band is used to limit the noise sequence [15]. The power spectrum of the simulated noisy signal is similar to the experimental VEP signal and it resembles the VEP recordings (Fig. 3(b)). Fig. 3(c) shows the results of ensemble averaging 10 ensembles of simulated noisy VEP signal. As what we expected, the SNR has been improved, however it is still hard to determine the latency and the amplitude of the peak. An adaptive neural network filter is used to filter the noisy VEP signals. The outputs from the filter after passing the $20^{\text {th }}$ ensemble are shown in Fig. 3(d). The VEP estimated by the adaptive neural network filter is very close to the actual underlying signal.

Further simulations are carried out to compare the performance of adaptive neural network filter to traditional linear adaptive filter (order $=20$ ). Different sets of simulation signal consisting of 100 ensembles of VEP signal with specific SNR, are generated by the method mentioned above. In each set, all ensembles are inputted to both filter and the SNR of their filtered signal is calculated. As shown in Fig. 4, the overall performance of adaptive neural network filter is better than the traditional linear adaptive filter especially when the SNR of noisy VEP signal is poor. The outstanding performance of adaptive neural network filter could be attributed to the hidden layer and the non-linear neuron transfer function, which are structured like a human brain.

\section{Conclusions}

An adaptive version of neural network filter is described for estimating the VEP signal. The most important feature of the proposed scheme is the connection weights are iteratively updated. During the filtering operation, the neural network keeps on learning in order to adapt to the non-stationary characteristic of the VEP signal. Simulation results confirm the successful operation of the filter. The results also show that the performance of adaptive neural network filter is superior to the linear adaptive filter and this may be accounted for by the non-linear characteristic of the neural network matching the nonlinear behaviour of VEP signal. The adaptive neural network filter enables VEP estimation from a singletrial input with much improved SNR and the observation of trial-to-trial variation, providing the clinician information regarding the variation of VEPs across trials.

\section{References}

[1] D. Regan, Evoked Potentials in Psychology, Sensory Physiology, and Clinical Medicine. New York: Wiley, 1972.

[2] M. J. Aminoff, Electrodiagnosis in Clinical Neurology. New York: Churchill Livingstone, 1980.

[3] T. Harmony, Neurometric Assessment of Brain Dysfunction in Neurological Patients. Hillsdale, NJ: Lawrence Erlbaum; 1984.

[4] D. Regan, Human Brain Electrophysiology: Evoked Potentials and Evoked Magnetic Fields in Science and Medicine. New York: Elsevier; 1989.

[5] C. D. McGillen, J. I. Aunon, K. B. Yu, "Signals and Noise in Evoked Brain Potentials". IEEE Trans. Biomed. Eng. BME-32: pp. 1012-1016; 1985.

[6] V. Albrecht, P. Lansky, M. Indra and T. RadilWeiss, "Wiener Filtration Versus Averaging of Evoked Responses", Biol. Cybern., vol. 27, pp.147-154, 1977.

[7] H. F. Li, F. H. Y. Chan, P. W. F. Poon, J. C. C. Hwang, W. S. Chan, "Maximum Length Sequence Applied to Brainstem Auditory Evoked Response (BAER)", Journal of Biomed. Engineering, vol. 10, pp. 14-24, Jan. 1988.

[8] S. Cerutti, G. Chiarenza, D. Liberati, P. Mascellani, and G. Pavesi, "A Parametric Method of Identification of Single-Trial EventRelated Potentials in the Brain", IEEE Trans. 35: pp. 701-711, 1988.

[9] F. H. Y. Chan, F. K. Lam, W. Qiu, P. W. F. Poon, "An Enhanced Approach to Adaptive Processing of the Brain Stem Auditory Evoked Potential", Australasian Physical and Engineering Sciences in Medicine, vol. 17, pp. 131-135, 1994.

[10] N. V. Thakor, "Adaptive Filtering of Evoked Potentials", IEEE Trans. Biomed. Eng., vol. 34, No. 1, pp. 6-12, 1987.

[11] F. K. Lam, F. H. Y. Chan, P. W. F. Poon and W. Qiu, "Visual Evoked Potential Measurement by Adaptive Filtering", Biomed. Materials and Engineering, vol. 4, No. 6, pp. 409-471, 1994.

[12] M. V. Shirvaikar, M. M. Trivedi, "A Neural Network Filter to Detect Small Targets in High Clutter Backgrounds", IEEE Trans. Neural Network, vol. 6, No. 1, pp. 252-257, 1995.

[13] R. H. Baran, J. P. Coughlin, "Neural Network for Passive Acoustic Discrimination between Surface and Submarine Targets", in Proc. Automat. Object Recogn. Conf. SPIE, Orlando, FL, Apr. 1991, pp. 164-176. 
[14] K. S. M. Fung, F. H. Y. Chan, F. K. Lam, P. W F. Poon, J. G. Liu, "Visual Evoked Potential Estimation by Artificial Neural Network Filter: Comparison with the Ensemble Averaging Method", Proc. IEEE International Conf. BME, Montreal, Sept. 1995 (in press).

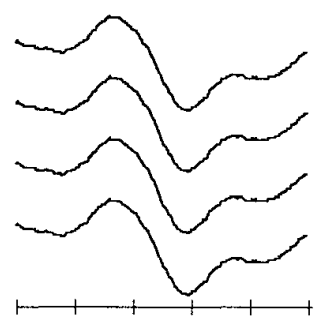

(a)

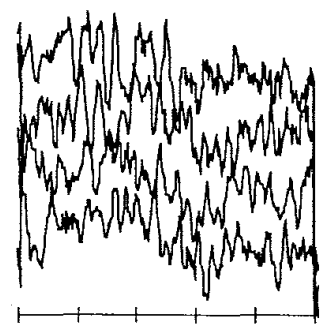

(b)
[15] X. H. Yu, Y. S. Zhang, Z. Y. He, "Peak Component Latency-Corrected Average Method for Evoked Potential Waveform Estimation", IEEE Trans. Biomed. Eng., vol. 41, No. 11, pp. 1072-1082, 1994.

Fig. 3: Results of simulations: (a) The underlying VEP signal. (b) Signal added with simulated noise. (c) Results of EA using 10 ensembles. (d) Result of a converged adaptive netral network filter using signals in (b) as noisy input signal.

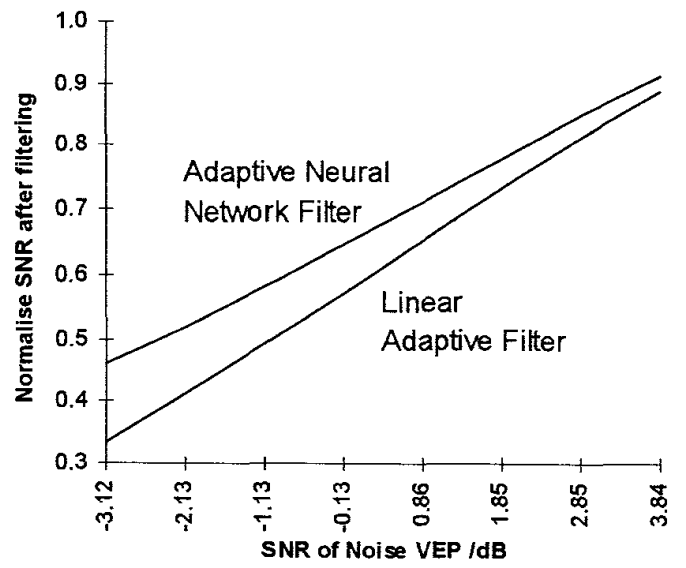

Fig. 4: The performance of linear adaptive filter and adaptive neural network filter is compared by feeding in noisy VEP with different $S N R$. 